\title{
Second Level Management of a Complex Case with Suspected Cardiac Amyloidosis: A Challenge for the Clinician
}

\author{
Valerio Massimo Magro* \\ Department of Internal Medicine and Geriatry, University of Campania Luigi Vanvitelli, Italy
}

\begin{abstract}
We report the clinical case report of a 67-year-old patient with diagnoses of hypertension, ischemic heart disease and a previous history of Acute Myocardial Infarction (AMI) and nonspecific signs and symptoms (general illness, weight loss, hyporexia, fever, impressive edema at the feet, systemic arterial hypotension). So, we performed a series of laboratory, radiological and instrumental examinations, but the clinical picture remained difficult to interpret. The echocardiographic evaluation led us to a suspicion of infiltrative heart disease, and the magnetic resonance, while confirming the interesting data observed at the ultrasound exam. Therefore, we decided to perform a biopsy, which appeared positive for our suspicion. So, we thought we did not waste any more precious time and, after stabilizing the patient, we sent our patient to a specialized diagnostic center for diagnostic confirmation and the beginning of targeted therapies. Clinical, laboratory and instrumental implications of this differential diagnosis are discussed.
\end{abstract}

\section{Keywords}

Differential diagnosis, Elderly patient, Amyloidosis, Heart failure, Echocardiography, Cardiac MRI

\section{Introduction}

The finding of a laboratory data or a sign of organ damage to the imaging exam is often classified as a consequence of the main diseases afflicting the Western World (arterial hypertension, ischemic heart disease, diabetes). In any case, the clinician must look into the decision-making process aimed at the clarification of a diagnostic suspect, from a whole series of pathologies that, even more rarely, can enter into a differential diagnosis. There are various infiltrative and degenerative processes in the heart representing a diagnostic challenge for a clinician. In fact, the diagnosis of these conditions can represent a challenge for the doctor in a non-specialized center, given the not frequent recourse to these patients. For example, cardiac localization of the amyloidotic degeneration is a rare and fearsome pathological condition, clinically and semasiologically not easy to recognize. It can manifest itself or as a complication of a systemic amyloidosis. In any case, cardiac involvement occurs in a cardiomegaly and subsequently, in a presence of a heart failure which is predominantly in diastolic phase in the early stages, while in the late stage it manifests itself as a restrictive-infiltrative cardiomyopathy with a contractile deficit and a fall of the ejection fraction (EF). So, cardiac involvement is associated either with heart failure with preserved ejection fraction (HFpEF), above all in the early stages, or with systolic dysfunction, usually in the late stage, expressing as a restrictive-infiltrative cardiomyopathy with a contractile deficit of the ejection fraction (HFrEF), with a severe prognosis. Here we describe a clinical case report, that came to our observation and that represented a diagnostic challenge. We studied using laboratory investigations and first and second level imaging techniques, trying to follow the recommendation of the scientific evidence.

\section{Case Presentation}

A 67-year-old patient with the diagnoses of hypertension, ischemic heart disease and a previous history of Acute Myocardial Infarction (AMI) treated with angioplasty and a stent implant for occlusion of the median tract of the anterior interventricular artery branch (IVA), came to our attention for the presence of general illness, a significant weight loss (about $20 \mathrm{~kg}$ in a period of about six months), hyporexia and lowgrade fever. The subject, in expired general conditions, was still vigilant, lucid, oriented and cooperative. The body Mass Index (BMI) was $18 \mathrm{~kg} / \mathrm{m}^{2}$. Vital signs were in the limits: Glasgow Coma Scale (GCS) $15(4+5+6)$, BP 110/70 mmHg (with anamnestic tendency to low pressure); HR $60 \mathrm{bpm}$, rhythmic; RR 19 breaths per minute; $\mathrm{SpO}_{2} 95 \%$ in the ambient air.

*Corresponding author: Valerio Massimo Magro, Geriatricians, M.D, Department of Internal Medicine and Geriatry, University of Campania Luigi Vanvitelli, Naples, Italy

Accepted: January 23, 2019

Published online: January 25, 2019

Citation: Magro VM (2019) Second Level Management of a Complex Case with Suspected Cardiac Amyloidosis: A Challenge for the Clinician. Ann Heart 4(1):74-78 
Citation: Magro VM (2019) Second Level Management of a Complex Case with Suspected Cardiac Amyloidosis: A Challenge for the Clinician. Ann Heart 4(1):74-78

\section{Management}

The clinical evaluation revealed the presence of faint cardiac tones in the presence of chest objectivity with normal semeilological parameters. Relaxed and treatable abdominal wall on all quadrants with evidence of intestinal limbs a lot expanses and hypertympanism. The alvus was normal, with slight contraction of the diuresis. Impressive edema at the feet and around malleolar and ankle regions. Hematochemical exams showed parameters in the limits except: WBC $2.8 \times$ $103 /$ ul (normal values 4.2-29), neutrophils $1.6 \times 103 /$ ul (2.2$4)$, lymphocytes $1 \times 103 /$ ul (1,3-2.9), monocytes $0.1 \times 103$ / ul (0.3-0.8), RBC $2.62 \times 106 / \mu \mathrm{l}$ (4.5-6.1), Hb $7.2 \mathrm{~g} / \mathrm{dl}(13-17)$, serum glucose $66 \mathrm{mg} / \mathrm{dl}(70-100)$, total protein $4.9 \mathrm{~g} / \mathrm{dl}(6.6-$ 8.7), albumin $3.1 \mathrm{~g} / \mathrm{dl}$ (3.5-5.5), potassium $2.7 \mathrm{mEq} / \mathrm{l}$ (3.5$5.3)$, total calcium $7.9 \mathrm{mg} / \mathrm{dl}$ (8.6-10.2), hypocolesterolemia. Immunoglobulin light chain were within the normal range (Figure 1). We also performed a bone marrow biopsy, but no plasma cell clones were found, so that in the absence of medullary blasts, we waited instead for a serum and urinary immunofixation. Anyway, urinary immunofixation confirmed the negative results of serum dosage. The esophagogastroduodenoscopy and colonoscopy did not show anything relevant except the presence of hiatal hernia

\begin{tabular}{lccc} 
CATENE KAPPA & 2.09 & $g / 2$ & $1.38-3.75$ \\
\hline CATENE LAMBDA & $* 0.84$ & $g /$ & $0.93-2.42$ \\
\hline RAPPORTO KL & 2.49 & $1.17-2.93$
\end{tabular}

Figure 1: Dosage of the light chains immunoglobulin. Urinary immunofixation confirmed the negative results of serum dosage.
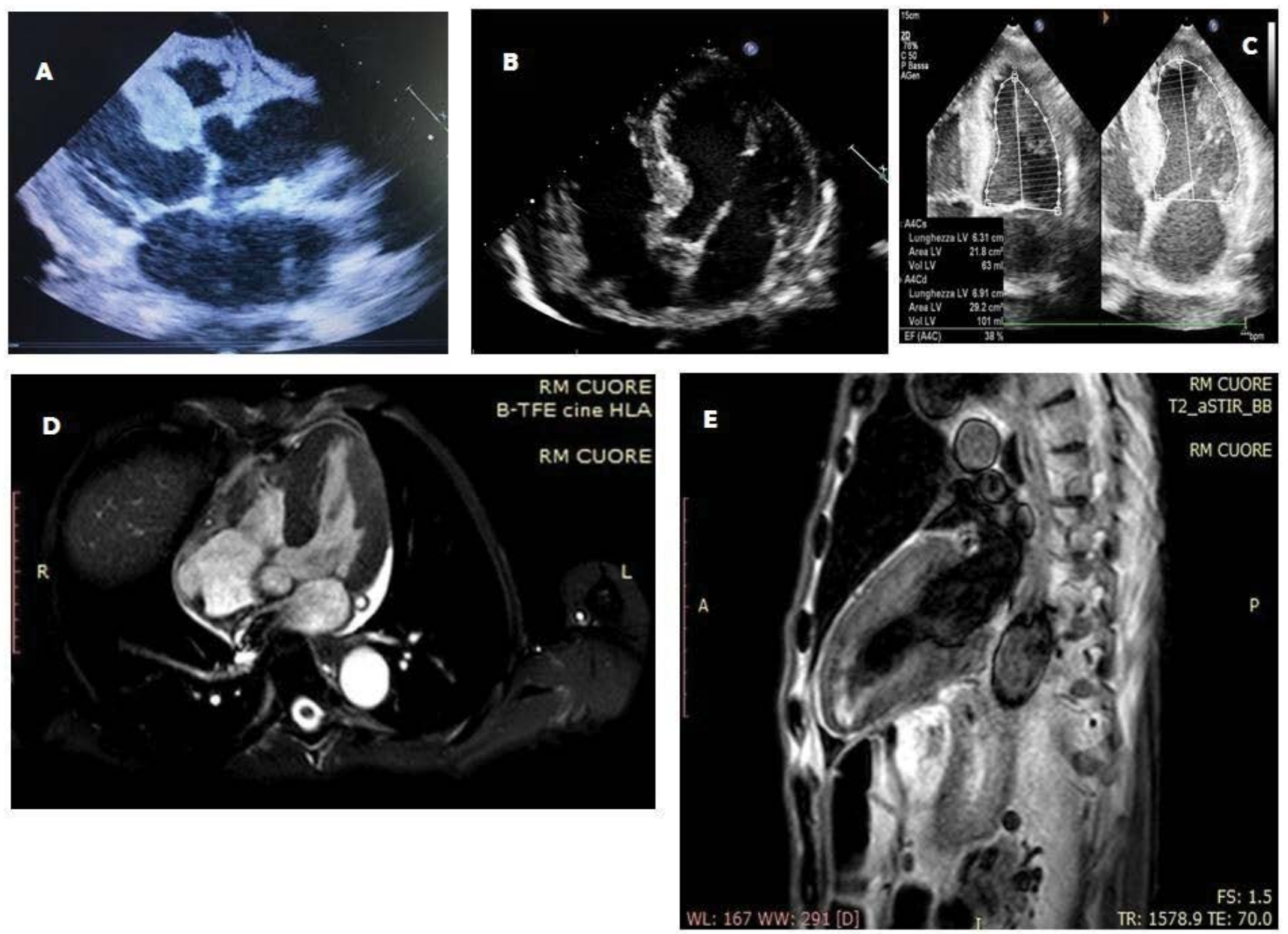

Figure 2: Transthoracic Echocardiography demonstrates increased LV mass and wall thickness A) Two-dimensional echo long-axis view showing LV hypertrophy and pericardial effusion. Dilated left atrium; B) Apical five chambers transthoracic echocardiography view showing the hypertrophy with the presence of a reduced LV global function (global hypokinesis and severely decreased cardiac function with an EF measured by Simpson's formula of 38\%; C) Granular sparkling of anterior interventricular septum and circumferential mild pericardial effusion. Cardiac-MRI confirming the LV concentric diffuse hypertrophy. A hyperechoic stria is also observed within the cardiac walls, with involvement of the interventricular septum up to the cardiac apex. Confirmed report on magnetic resonance. Unfortunately, there was lack of late gadolinium enhancement; $D, E$ ) The strong diagnostic suspicion was increased by the positive results obtained by biopsy. 
associated with a grade A erosive esophagitis according to Los Angeles staging. The EKG showed a sinus bradycardia with HR $58 \mathrm{bpm}, \mathrm{PQ} 0.20 \mathrm{~s}$, QRS $0.06 \mathrm{~s}$, presence of $\mathrm{Q}$ waves from $\mathrm{V} 1$ to V4 and inversion of T waves in V2-V3. At this point of management an echocardiogram was performed: it showed an increased LV mass and wall thickness with LV hypertrophy and pericardial effusion, left atrial enlargement, reduced LV global function ( $E F<50 \%$ ), increased echo-reflective of the ventricular myocardium. These findings were confirmed by the execution of a cardiac-MRI (Figure 2, respectively A-C and D-E). Immunologic exams (ANAs, ENAs antibodies), oncomarkers, emocultures and serology for Coxsackievirus, HSV 1 and 2, EBV, HBV, HCV, Echo, Parvovirus B19, Influenza, HIV and Toxoplasma were negative, while Quantiferon TB Gold highlighted an increased levels of gamma interferon after stimulation with specific antigens for M.Tuberculosis $(1.71 \mathrm{UI} / \mathrm{ml}$; cut-off 0.35$)$ and with mitogen (> $10 \mathrm{UI} / \mathrm{ml}$; cutoff 0.50 ). We suspected a myocardial storage disease but the dosage of the enzyme of Fabry's disease turned out to be negative and, due to technical problems, it was not possible to organize the execution of a scintigraphy. Then, we performed an excision biopsy of peri-umbilical fat, detecting, in the coloration with the red congo dye, the presence of amorphous orangiophilic material in the context of the fibrous tissue, which at the observation in the polarized light microscope assumed a green apple coloration, found compatible with the deposition of the amyloid substance. Thus, the patient was subjected at a total body computhed tomography (CT), that, while excluding a concomitant pathology of neoplastic nature, showed a hypodense formation at the pulmonary level deserving for further diagnostic research to determine its nature (suspected tuberculosis or an amyloid pulmonary accumulation). Thus, we started an energetic diuretic therapy with the optimization of the diuresis (approximately $1500 \mathrm{cc}$ ), the drainage of the pericardial effusion fluid, the improvement of sloping edema and general status of the patient, the albuminemic reintegration (with a mild and temporary albumin supplementation, also in order to optimize the loop diuretic therapy, bringing the dosage of furosemide up to $500 \mathrm{mg}$ and then we proceed with the down-titration) and the electrolyte balance ( $\mathrm{KCl}$ and calcium gluconate), we also improved the patient anemic status (latest hemoglobin rises to $8.6 \mathrm{~g} / \mathrm{dl}$ after folate and vitamin B12 therapy), while we set the concomitant cardiologic and gastroenterological therapy (valsartan $160 \mathrm{mg}$, hydrochlorothiazide $12.5 \mathrm{mg}$, acetylsalicylic acid (ASA) $100 \mathrm{mg}$, clopidogrel $75 \mathrm{mg}$, atorvastatin $80 \mathrm{mg}$, pantoprazole $20 \mathrm{mg}$ ), finally for transferring the patient to a specialist amyloidosis center (third level center) for the further investigations (bronchoscopy, endomycardial biopsy, genetic tests) and any therapies of this case.

\section{Discussion}

This case report turned out to be very interesting because it has put us in a position to seld-audit our skills and kwonledge related to the management of patients with infiltrative organ or systemic pathology such as amyloidosis or other substances that cause a severe dysfuncions organ. Amyloidosis is precisely an uncommon, severe prognosis, condition which consists in the deposition of an abnormal protein, amyloid, in the extracellular space of different organs and tissues. This protein material is arranged to form a fibrillary structure that is specifically colored with red congo dye or with a color-based hematoxylin-eosin or it is observed in polarized light microscope (by which it assumes a greenapple color) and it is obtainable from a sample by a needle aspiration from the peri-umbilical or skin adipose tissue and subjected to biopsy [1]. A localization which deals with peculiar clinical pictures, is at the level of the cardiac site [2]. Such localization may occur either as a secondary manifestation of a systemic amyloidosis or as an isolated expression of myocardial infiltration. Among the most prevalent cardiac localization forms is the systemic senile amyloidosis [3]. Outside of systemic and localized forms, secondary or reactive amyloidosis, including tuberculosis $[4,5]$ or some neoplasms [6-9], that should be mentioned. The initial symptoms of amyloidosis are quite nuanced and totally unspecific (weight loss, asthenia, dyspnoea, edema, difficulty in swallowing, sense of satiety). The most significant difficulty in diagnosing the cardiac localization is its late clinical presentation (often after the fifth decade of life) and the wide clinical variability. It manifests itself in the form of a rather severe restrictiveinfiltrative cardiomyopathy [10-11]. A serum monoclonal component is detectable in a significant percentage of patients (identifying that particular type of amyloidosis in which the deposits are characterized precisely by light chains of the immunoglobulins - light-chain amyloidosis, LA - and in which there is often a cardiac involvment). In our case, this monoclonal component was not found. As for imaging, amyloid-involved myocardial walls show ultrasound image texture alterations that may be quantified with digital image analysis techniques. In the first place, cardiac amyloidosis can be recognized by echocardiographic examination. In fact, the mono- and bi-dimensional echocardiographic examination lays for an infiltrative myocardial disease: this condition is deduced from the left ventricular wall hypertrophic aspect to mono- and bi-dimensional echocardiographic investigation. The walls are also characterized by a typical "granular sparkling" (present in our case report) depending on the deposition of the abnormal protein in interstitial space [12]. Other echocardiographic signs include a dilation of the two atrial cavities, as well as frequent pericardial permeability" (pericardial effusion) [13]. Koyama J, et al. [14] describe some echocardiographic indices are prognostic for the amyloidoses, with M-mode and 2-dimensional echocardiography (such as the left ventricular - LV - wall thickness) and Doppler flow measurements (as the peak flow velocity and acceleration rate of early diastolic filling and the ratio of atrial peak flow velocity/early diastolic peak flow velocity or the pulmonary vein peak systolic flow). Mothy $D$, et al. [3] mentioned other parameters still associated with poor prognosis, such as the left atrial enlargement and the increased right ventricle free wall thickness, the reduced aortic ejection time. new acquisitions on the subject of prognostic elements come more recently from echocardiographic studies that have used the speckle tracking method [15]. Cardiac-MRI has several properties in the evaluation of suspected amyloid heart disease: High spatial resolution that permit a reproducible measurement of cardiac chamber volumes and mass, as 
Citation: Magro VM (2019) Second Level Management of a Complex Case with Suspected Cardiac Amyloidosis: A Challenge for the Clinician. Ann Heart 4(1):74-78

well as LV and atrial septal wall thickness. It can characterize pericardial and pleural fluid, too [16]. Furthermore, the use of the contrast agent (gadolinium) can provide more valuable information (such as the presence of a diffuse, subendocardial late enhancement, mainly in the LV wall (not present here), or the accelerated gadolinium kinetics in blood and myocardium) [17-19]. A recent meta-analysis of Zhao $\mathrm{L}$, et al. concerning the use of gadolinium concluded that its late enhancement is more accurate in the diagnosis of cardiac amyloidosis, which makes it valuable in the detection of amyloid deposit although with different limitations in the application in clinical practice (claustrophobia, implanted cardiac devices, renal failure) [20]. Anyway, cardiac MRI also may help to estimate the prevalence of cardiac involvement in systemic amyloidosis when cardiac morphological changes are not apparent by echocardiography [18]. A consensus was recently published by the most important scientific societies that deal with the problem (the European Association of Cardiovascular Imaging, EACVI; the Working Group on Myocardial and Pericardial diseases of the European Society of Cardiology, ESC). In this document reaffirms the concept that reaffirms the addition of parametric mapping to standard cardiac MRI images is a powerful and quantitative diagnostic tool that also allows differential diagnosis from other diseases with similar phenotypic expression. Echocardiography and cardiac MRI constitute, together with the clinic, the first step in the diagnostic algorithm for patients with suspected amyloid cardiomyopathy [21]. The next step is the biopsy for amyloid protein investigation. Tissue samples usually undergo red congo staining. It enables obtaining pathognomonic green birefringence of stained tissues examined under the polarization light in the tissue of the presence of amyloid deposits in the tissue specimen [22]. There is a poor interobserver reproducibility with false negatives and positives. The false positivity is due to superficial resemblance of focal blue birefringence associated with collagen tissue with apple green birefringence of amyloid and it may be confirmed further by electron microscopy to overcome the disparity related to the inter-observer variation with red congo stained sections for detection of amyloid. Inconsistency related to the focal blue birefringence of collagen fibers in sections may be more challenging under routine surgical pathology-cytopathology setting with additional unanticipated factors such as limited understanding of uncommonly used polarizing microscopy [23]. For these reasons, in some cases it may be necessary to perform biopsies from other mucosa and/or in affected organs. Tuberculosis can be a common cause of amyloidosis in adults and our patient, besides being a cardiovascular patient, had both clinical and laboratory signs that led us to that suspected diagnosis, so we thought it was right to perform a bronchoscopy, both to confirm the suspicion of infectious disease and to carry out a histological assessment also to the lung to look for traces of amyloid $[5,24]$. Unfortunately we do not have other informations about the final diagnosis of our case report (AL vs. ATTRm vs. ATTRwt amyloidosis) but we believe it is right to launch the take home message that clinical cases like this should be taken in charge by third level centers, with greater experience in such cases, laboratories and technologies of the highest level and the possibility of subjecting patients to also experimental therapies $[25,26]$.

\section{Conclusions}

With the differential diagnosis a physician can consider the possible causes of patients' clinical findings before making a final diagnoses. The evaluation of a person with important but common comorbidities should not distract the clinician from being able to suspect the coexistence of other less common but certainly not less important pathologies: for example, cardiac amyloidosis refers to an infiltrative disease with poor prognosis that relies on identification of the amyloid type. In this disease, imaging techniques play a central role. Echocardiography and cardiac MRI techniques are of utmost value for the diagnostic and prognostic assessment of this condition. Imaging study findings and the key "red flags" a clinician should evaluate to avoid underdiagnosis of this important pathological condition and the doctor can also use higher-level diagnostic tests if the clinical picture remains incomplete. Also, tuberculosis is a pathology still difficult to recognize clinically in populations with a good standard of living such as the Italian. Anyway, the scenario patient's history suggests a multiple pathologic causes.

The described case report exhorts the clinician to leave open the way to suspected diagnoses of more rare pathologies rather than to direct the investigations only on the most common pathologies, if the evidence suggests, in order not to leave out even important hypotheses, sending to the specialized centers cases with high suspect index.

\section{Ethical approval}

"All procedures performed in studies involving human participants were in accordance with the ethical standards of the institutional and/or national research committee and with the 1964 Helsinki declaration and its later amendments or comparable ethical standards".

\section{References}

1. Donnelly JP, Hanna M (2017) Cardiac amyloidosis: An update on diagnosis and treatment. Cleve Clin J Med 84: 12-26.

2. Bodard Q, Roca F, Dilly B, et al. (2016) Acute cardiac failure secondary to senile systemic amyloidosis. Age Ageing 45: 908909.

3. Mohty D, Damy T, Cosnay P, et al. (2013) Cardiac amyloidosis: Updates in diagnosis and management. Arch Cardiovasc Dis 106: 528-540.

4. Balwani MR, Kute VB, Shah PR, et al. (2015) Secondary renal amyloidosis in a patient of pulmonary tuberculosis and common variable immunodeficiency. J Nephropharmacol 4: 69-71.

5. Fekih L, Boussoffara L, Fenniche S, et al. (2011) Enigmatic evolution of an association of pulmonary tuberculosis and amyloidosis. Rev Mal Respir 28: 691-695.

6. Nobata H, Suga N, Itoh A, et al. (2012) Systemic AA amyloidosis in a patient with lung metastasis from renal cell carcinoma. Amyloid 19: 197-200.

7. Bestard Matamoros $\mathrm{O}$, Poveda Monje R, Ibernon Vilaró M, et al. (2008) Systemic AA amyloidosis induced by benign neoplasms. Nefrologia 28: 93-98. 
8. Yamane T, Nakamura M, Uchiyama $K$, et al. (2005) A case of secondary amyloidosis from multiple myeloma with stenosis of the upper digestive tract. Nihon Shokakibyo Gakkai Zasshi 102: 1176-1182.

9. Onishi S, Hojo N, Sakai I, et al. (2005) Secondary amyloidosis and eosinophilia in a patient with uterine leiomyosarcoma. Jpn J Clin Oncol 35: 617-621.

10. Karafiatovaa L, Pikab T (2017) Amyloid cardiomyopathy. Biomed Pap Med Fac Univ Palacky Olomouc Czech Repub 161: 117-127.

11. Patel KS, Hawkins PN (2015) Cardiac amyloidosis: Where are we today? J Intern Med 278: 126-144.

12. Pinamonti B, Picano E, Ferdeghini EM, et al. (1989) Quantitative texture analysis in two-dimensional echocardiography: Application to the diagnosis of myocardial amyloidosis. J Am Coll Cardiol 14: 666-671.

13. Mesquita ET, Jorge AJL, Souza CV Junior, et al. (2017) Cardiac Amyloidosis and its New Clinical Phenotype: Heart Failure with Preserved Ejection Fraction. Arq Bras Cardiol 109: 71-80.

14. Koyama J, Ikeda S, Ikeda U (2015) Echocardiographic assessment of the cardiac amyloidoses. Circ J 79: 721-734.

15. Phelan D, Collier P, Thavendiranathan $P$, et al. (2012) Relative apical sparing of longitudinal strain using two-dimensional speckletracking echocardiography is both sensitive and specific for the diagnosis of cardiac amyloidosis. Heart 98: 1442-1448.

16. Selvanayagam JB, Leong DP (2010) MR Imaging and Cardiac Amyloidosis Where to Go From Here? JACC Cardiovasc Imaging 3: $165-167$.

17. Domínguez A, Garrido N, Maceira A (2005) Cardiac magnetic resonance imaging in amyloidosis. Rev Esp Cardiol 58: 1132-1133.

18. Maceira AM, Joshi J, Prasad SK, et al. (2005) Cardiovascular magnetic resonance in cardiac amyloidosis. Circulation 111: 186-193.
19. Divakaran S, Singh A, Collins B, et al. (2017) Role of Imaging in Evaluating Infiltrative Heart Disease. Curr Treat Options Cardiovasc Med 19: 3.

20. Zhao L, Tian Z, Fang Q (2016) Diagnostic accuracy of cardiovascular magnetic resonance for patients with suspected cardiacamyloidosis: A systematic review and meta-analysis. BMC Cardiovasc Disord 16: 129.

21. Habib G, Bucciarelli-Ducci C, Caforio ALP, et al. (2017) Multimodality Imaging in Restrictive Cardiomyopathies: An EACVI expert consensus document In collaboration with the "Working Group onmyocardial and pericardial diseases" of the European Society of Cardiology Endorsed by The Indian Academy of Echocardiography. Eur Heart J Cardiovasc Imaging 18: 1090-1121.

22. Jerzykowska S, Cymerys M, Gil LA, et al. (2014) Primary systemic amyloidosis as a real diagnostic challenge - case study. Cent Eur J Immunol 39: 61-66.

23. Devata S, Hari P, Markelova N, et al. (2011) Detection of amyloid in abdominal fat pad aspirates in early amyloidosis: Role of electron microscopy and Congo red stained cell block sections. Cytojournal 8: 11.

24. le Roux DM, Pillay K, Nourse P, et al. (2012) Systemic Amyloidosis Complicating Multidrug-Resistant Tuberculosis in Childhood. Pediatr Infect Dis J 31: 994-997.

25. Ramaekers J, Janssens J, Waumans L, et al. (2019) Indications and diagnostic yield of endomyocardial biopsies for unexplained cardiomyopathy, a single center experience. Acta Cardiol 16: 1-9.

26. Gupta VK, Brauneis D, Shelton AC, et al. (2019) Induction Therapy with Bortezomib and Dexamethasone and Conditioning with High-Dose Melphalan and Bortezomib Followed by Autologous Stem Cell Transplantation for AL Amyloidosis: Long Term FollowUp Analysis. Biol Blood Marrow Transplant.

DOI: $10.36959 / 652 / 396$

Copyright: (C) 2019 Magro VM. This is an open-access article distributed under the terms of the Creative Commons Attribution License, which permits unrestricted use, distribution, and reproduction in any medium, provided the original author and source are credited. 